\title{
Proprioceptive neuromuscular facilitation (PNF) vs. task specific training in acute stroke: the effects on neuroplasticity
}

\begin{abstract}
Objectives: There are several approaches for rehabilitation of stroke patients. Proprioceptive Neuromuscular Facilitation (PNF) and Task Specific Training are the two approaches, known to promote motor learning and motor control. In this study we aim to compare both approaches on the basis of Brain Derived Neurotrophic Factor (BDNF) levels in the serum before and after intervention and at 6 months.

Methods: 90 subjects were recruited in this study. Subjects were divided into two groups. Group 1 was given PNF exercises and group 2 was given task specific training. Both group received intervention 30 minutes twice daily, five days a week for four weeks.

Results: PNF group showed more improvement than task specific group. There was significant improvement in serum BDNF levels $(p=.004)$, FMA scores $(p=.003)$ at 4 weeks and at 6 months (S. BDNF $\mathrm{p}=.001, \mathrm{FMA} \mathrm{p}=<.05)$. Group 1 showed $53.02 \pm 34.14$ points and group 2 showed $38.82 \pm 40.24$ points, which is significant. There was $5.89 \pm 4.07 \mathrm{ng} / \mathrm{ml}$ raise in S.BDNF in group 1 and $3.45 \pm 4.19 \mathrm{ng} / \mathrm{ml}$ in group 2 from admission to 6 months. Results are showing more improvement in PNF group.

Conclusion: PNF exercises may effective in promoting neuroplasticity and functional activities. They are more effective if implemented as early as possible. On the basis of finding of our study PNF can be recommended as standardized approach of rehabilitation in acute stroke if the patient is able to follow the commands.
\end{abstract}

Keywords: acute stroke, proprioceptive neuromuscular facilitation, task specific training, BDNF, fugl-meyer assessment
Volume 5 Issue 2 - 2018

\author{
Poonam Chaturvedi,' Ajai Kumar Singh,' \\ Vandana Tiwari, ${ }^{2}$ Dinkar Kulshreshtha,' \\ Pradeep Kumar Maurya,' Anup Kumar \\ Thacker' $^{\text {Th }}$ \\ 'Department of Neurology, Dr. Ram Manohar Lohia Institute of \\ Medical Sciences, India \\ 2Department of Biochemistry, Dr. Ram Manohar Lohia Institute \\ of Medical Sciences, India
}

\section{Correspondence: Poonam Chaturvedi, Department of Neurology, Dr. Ram Manohar Lohia Institute of Medical Sciences, Lucknow, India, 226010 \\ Email poonamchaturvediphysio@gmail.com}

Received: February 13, 2018 | Published: April 24, 2018

\section{Introduction}

Stroke is the major cause of disability. Disability associated with hemiplegia or hemiparesis markedly limits the independent living and social participation in at least half of all stroke survivors. ${ }^{1}$ Recovery of motor function after stroke involves relearning motor skills and is mediated by neuroplasticity. Although many molecular signalling pathways are involved, brain-derived neurotrophic factor (BDNF) has emerged as a key facilitator of neuroplasticity involved in motor learning and rehabilitation after stroke. ${ }^{2}$ Recent research has focused on developing rehabilitation strategies that facilitate such neuroplasticity to maximize functional outcome post stroke. A variety of neurologically based techniques are used by physical therapists in the treatment of hemiplegic patients. Although these techniques are used widely, few studies have been reported in the literature validating these diverse approaches for specific conditions or problems. Proprioceptive Neuromuscular Facilitation (PNF) is a philosophy of treatment based on principles of neurophysiology. Kabat ${ }^{3,4}$ suggested that patterns of movements performed in combination with other facilitatory procedures result in enhanced voluntary responses. The PNF approach to treatment uses the principle (based on early phylogenetic and embryologic observations that control of motion proceeds from proximal to distal body regions. Facilitation of trunk control, therefore, is used to influence the extremities. ${ }^{3-8}$ Studies reported PNF intervention in subacute and chronic stroke. Studies to the best of our knowledge regarding PNF implementation in acute stroke and its effects on neuroplasticity are still lacking.
On the other side a task-oriented exercise program as a new strategy focuses on functional retraining in subjects with stroke by using multi-system interactions, including the musculoskeletal, cognitive, and neurological systems. ${ }^{9-11}$ Task oriented exercise focuses on individual's goals and personal needs; and using verbal and visual feedback during practice., ${ }^{9,12,13}$

In this study we aim to compare the effects of two therapies on neuroplasticity (S. BDNF) and sensory- motor recovery in patients with acute stroke.

\section{Subjects and methods}

\section{Subjects}

90 subjects who were admitted to our hospital were recruited in this study after providing written informed consent. The study was approved by institutional ethical committee. Subjects were recruited into the study having first ever stroke, diagnosed by neurophysician on the basis of clinical and neuroimaging findings (CT, MRI), between age 40-60 years, having first time stroke. Patients were assessed for consciousness and orientation by Glasgow Coma Scale, stroke severity by NIHSS and functional level by Modified Rankin Scale. Sensory motor recovery was checked by Fugl-Meyer Scale. Subjects were divided into two groups. Group 1 was given PNF exercises and subjects in group 2 were given task specific training for upper and lower extremity. BDNF levels were assessed before and after the intervention of 4 weeks in both groups. Patients were discharged to 
home along with the advice to continue the exercises at home along with the prescribed medication and followed up in OPD on monthly basis up to 6 months to assess further improvement or any adverse event.

\section{Inclusion and exclusion criteria}

The patients included having first time stroke, between the age of 40-70 years. Patients excluded from the study who were having recurrent stroke, aphasia, severe cardiac illness (MI), fracture, pregnancy, NIHSS $>20, \quad M M S E<19$, any psychiatric illness, subarachnoid hemorrhage, amputation.

\section{Intervention}

\section{PNF exercises}

PNF exercises were implemented to group 1 in cephalo-caudal direction. Initially the exercises were given for neck and trunk followed by scapula, pelvis and then upper and lower extremity.

PNF for neck: Flexion with rotation to the left and extension with rotation to the right and vise-Versa.

PNF for trunk: Rhythmic stabilization and alternating isometrics.

PNF for scapula and pelvis: Anterior elevation and posterior depression; posterior elevation and anterior depression by rhythmic initiation and repeated contraction.

For upper and lower extremity: D1 and D2 flexion and extension patterns

\section{Task specific training}

In group 2, the patients performed the following task-oriented exercise program for upper and lower extremity: 1) sitting on a chair and reaching for objects in all directions at a distance of more than arm's length, 2) stepping forward, backward, and sideways on the exercise step, 3) performing flexion and extension of the affected knee, with the affected foot located on the exercise step and the unaffected foot off the step, 4) stepping over obstacles with different heights, 5) standing up from a chair, walking four steps forward, touching a stool, and then returning to the chair with support, 6) sitting on a Swiss ball while doing a range of motion and balance exercises in the trunk and upper extremities with support, 7) double leg standing for 10 seconds, 8) tandem standing, or placing the heel of one foot in front of the other foot, for 10 seconds, 9) standing up from a chair without using the arms, and 10) tandem walking forward and backward with support. The four last exercises were performed in different situations while the somatosensory and vision were manipulated as follows: 1) with open eyes and a hard surface, 2) with open eyes and a soft surface, 3 ) with closed eyes and a hard surface, and 4) with closed eyes and a soft surface. The difficulty level of the exercises was determined by increasing the number of repetitions. ${ }^{14}$ Both groups received the intervention for 30 minutes twice daily, five days a week for four weeks.

\section{Estimation of brain derived neurotrophic factor}

Assessment of serum BDNF levels were done by Enzyme Linked Immunosorbent Essay (ELISA). $5 \mathrm{ml}$ of blood was drawn from anticubital vein from each subject. Blood was centrifuged and serum was separated and stored at $-80^{\circ} \mathrm{C}$. We used commercial ELISA KIT (Ray Biomed Human BDNF ELISA kit). ELISA was run as per kit protocol at $37^{\circ} \mathrm{C}$. BDNF levels were assessed by reading the O.D. absorbance at $450 \mathrm{~nm}$ within 10 minutes after adding the stop solution (Figure 1).

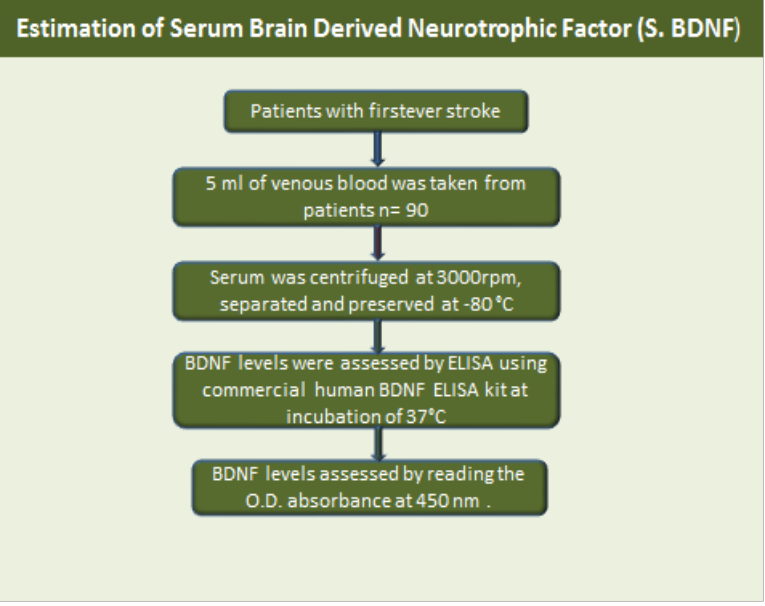

Figure I Showing the procedure followed for estimation of serum brain derived neurotrophic factor.

We used statistical package of social science (SPSS) version 20.0. The normality of data was checked by Kolmogorov Smirnov Test. We applied chi-square test to compare the categorical variables in both groups for baseline data and independent t-test to compare the continuous variables. We applied independent t-test to assess the difference in means in both groups and paired t-test to assess the improvement within group. A p value of $<.05$ was considered significant. Paired t-test was applied to compare the mean within group.

\section{Results}

The scores in our data was normally distributed $(\mathrm{p}=.890)$. There was no any significant difference in baseline characteristics in both groups (Table 1).

Table I Baseline characteristics of subjects

\begin{tabular}{|c|c|c|c|}
\hline & $\begin{array}{l}\text { Group I } \\
(n=49)\end{array}$ & $\begin{array}{l}\text { Group } 2 \\
(n=4 I)\end{array}$ & $\begin{array}{l}P \\
\text { value }\end{array}$ \\
\hline Age(yrs.) & $58.14 \pm 11.39$ & $61.29 \pm 12.56$ & $.86^{\mathrm{a}}$ \\
\hline Weight (Kg) & $64.60 \pm 14.62$ & $71.60 \pm 17.38$ & $.76^{\mathrm{a}}$ \\
\hline Gender (male/female) & $29 / 20$ & $28 / 13$ & $.21^{\mathrm{b}}$ \\
\hline $\begin{array}{l}\text { Side of stroke (left/ } \\
\text { right) }\end{array}$ & $30 / 19$ & $25 / 16$ & $.13^{\mathrm{b}}$ \\
\hline NIHSS & $6.53 \pm 3.79$ & $6.65 \pm 3.49$ & $.99^{a}$ \\
\hline $\begin{array}{l}\text { Type of stroke } \\
\text { (ischemic/hemorrhagic) }\end{array}$ & $30 / 19$ & $30 / 11$ & $>.99^{b}$ \\
\hline $\begin{array}{l}\text { Time since } \\
\text { stroke(days) }\end{array}$ & $30.29 \pm 9.14$ & $30.57 \pm 10.32$ & $.76^{a}$ \\
\hline MRS & $3.75 \pm 0.56$ & $3.68 \pm 0.75$ & $.87^{\mathrm{a}}$ \\
\hline
\end{tabular}

NIHSS, national institute of health stroke scale; MRS, modified rankin scale; a, independent $\mathrm{t}$ - test; ${ }^{\mathrm{b}}$, chi- square test

\section{Fugl-meyer assessment}

There was significant improvement in Fugl-Meyer score (Figure 
2) in both within and in between groups (Table 2). Group 1 showed $53.02 \pm 34.14$ points and group 2 showed $38.82 \pm 40.24$ points increase in the Fugl-Meyer scores (Figure 2) at 6 months from the baseline $\mathrm{t}(88)=-.30$ (Table 2). $\mathrm{P}=<.05$, which is quite significant.
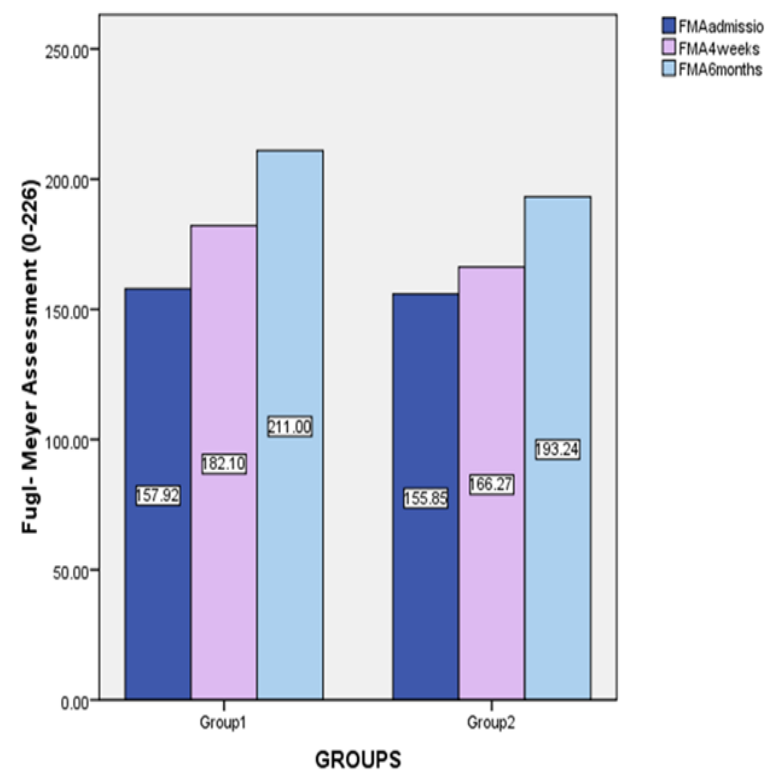

Figure 2 Showing rise in sensory motor scores in both groups with significantly more in PNF group.

Table 2 Comparison of BDNF and Fugl-Meyer scores in between groups

\begin{tabular}{lllll}
\hline Variables & $\begin{array}{l}\text { Experimental } \\
\text { group }\end{array}$ & $\begin{array}{l}\text { Control } \\
\text { group }\end{array}$ & P-value & $95 \% \mathbf{C l}$ \\
\hline BDNF(ng/mI) & & & \\
Admission & $9.35 \pm 3.75$ & $8.75 \pm 4.17$ & $0.476^{*}$ & $-1.06-2.26$ \\
4Weeks & $12.14 \pm 3.16$ & $10.33 \pm 3.35$ & $0.004^{*}$ & $0.500-3.20$ \\
6 months & $14.86 \pm 3.20$ & $12.03 \pm 4.30$ & $0.001 *$ & $1.25-4.40$ \\
FMA & & & & \\
Admission & $157.92 \pm 42.8$ & $155.85 \pm 44.96$ & $0.824 *$ & $-16.36-$ \\
4 Weeks & $182.10 \pm 38.13$ & $166.27 \pm 30.03$ & $0.003 *$ & $1.24-30.42$ \\
6 months & $211.00 \pm 22.84$ & $193.24 \pm 17.98$ & $<0.05$ & $9.01-26.49$ \\
\hline
\end{tabular}

*Independent t- test

\section{Serum brain derived neurotrophic factor}

Rise in serum BDNF levels from baseline to 6y months were there in both groups. Serum BDNF levels (Figure 3) within group. There was $5.89 \pm 4.07 \mathrm{ng} / \mathrm{ml}$ raise in S.BDNF in group 1 and $3.45 \pm 4.19 \mathrm{ng} / \mathrm{ml}$ in group 2 from admission to 6 months. On comparing both groups, the PNF group showed more improvement in BDNF levels (Figure 3) as compared to the group received task specific training $\mathrm{t}(88)=0.732$ $\mathrm{p}=<.05$ after intervention.
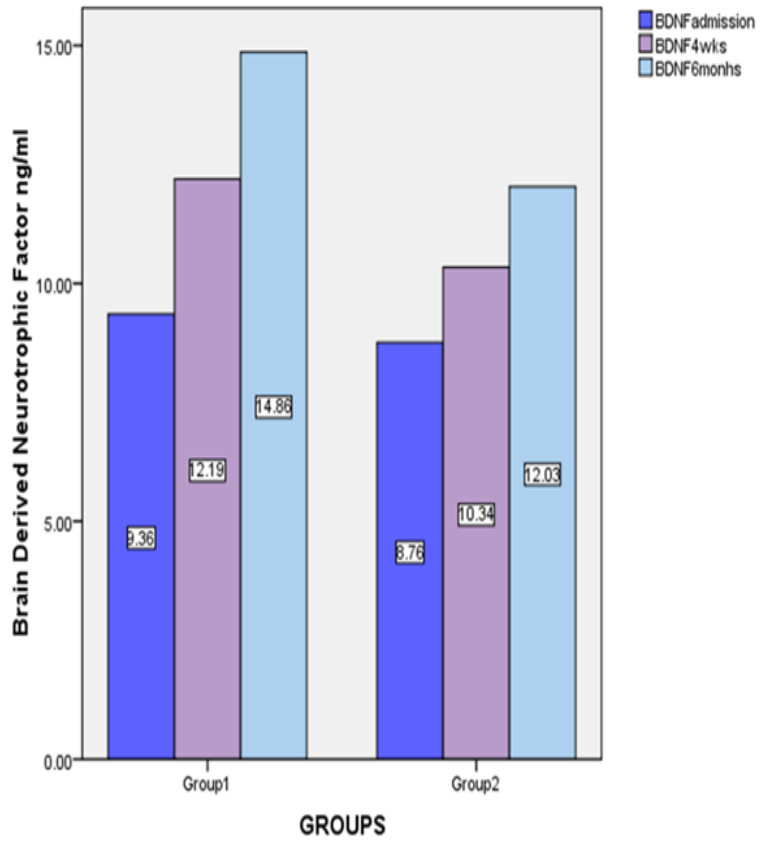

Figure 3 Showing rise in serum levels of BDNF in both groups with significantly more in PNF group.

\section{Discussion}

The main objective of our study was to know the technique that is more effective in promoting neuroplasticity. In this study we have compared two techniques of rehabilitation in stroke patients. PNF is an intensive exercise. On the other hand repeated training of specific task leads to permanent learning of task and brain reorganization which is also termed as neuroplasticity. But the question is how we come to know which intervention is better. The answer is we can assess the levels of BDNF before and after intervention. The approach that increases the BDNF levels in serum can be a better approach. Positive effects of early PNF exercises on functional outcome and quality of life in patients with acute stroke motivated us to assess the effects of PNF exercises on neuroplasticity also. ${ }^{15}$

Brain-derived neurotrophic factor (BDNF), the most abundant neurotrophin within the brain, is important for post stroke recovery, since it promotes neurogenesis and angiogenesis in animals. ${ }^{16,17}$ $\mathrm{BDNF}$ is stored and released from glutamatergic neurons in a use dependent fashion and has been implicated in long term potentiation, learning, memory formation, depression and recovery from brain injury. ${ }^{18}$ Circulating BDNF protein levels are lowered in acute phase of stroke, and low levels are associated with poor long term functional outcome. ${ }^{19}$ In a study Stanne et al. ${ }^{19}$ has demonstrated that BDNF levels were lower in stroke patients as compared to the healthy controls. Studies in the current time are showing more interest in the neuroplasticity. Stroke rehabilitation must be such that which can raise the neurotrophin level such as BDNF, Nerve Growth Factor (NGF), GDNF, VEGF etc. As PNF work on the principle that repeated commands, traction, approximation, audiovisual cueing and stretch reflex is used to facilitate the impulses along the reflex arch to 
elicit the sensory motor response. So we assessed the sensory motor recovery by Fugl-Meyer Scale. In our study both groups showed improvement in BDNF levels and Fugl-Meyer Scores but PNF group showed more improvement than control group. According to Ferris et al. ${ }^{20}$ in healthy humans, short-term exercise increases the circulating BDNF level. ${ }^{20}$ Release of S. BDNF is also depends upon the intensity of exercise. A study carried out by Jeon et al. ${ }^{21}$ on forty male students concluded that high intensity exercises raises S. BDNF levels at rest. These results also favor our study. PNF exercises are also intensive exercises because there is use of resistance. This may be the reason of more rises in S. BDNF levels. This supports findings of RojasVega et al. ${ }^{22}$ who reported that when athletes performed $10 \mathrm{~min}$ of warm-up exercises, there was no notable change in the serum BDNF concentration level. However, after subjects performed ramp tests, the level of serum BDNF significantly increased. Also, Ferris et al. ${ }^{20}$ reported that when subjects performed 30 min of cycling at below $20 \%$ of threshold concentration level of ventilation ( $55 \% \mathrm{VO} 2 \mathrm{max})$, there was no notable difference in their serum BDNF level; however, when they performed $30 \mathrm{~min}$ of cycling at higher than $10 \%$ of threshold concentration level of ventilation ( $75 \% \mathrm{VO} 2 \mathrm{max})$, their serum BDNF level significantly increased.

Furthermore, several studies have reported a high serum BDNF concentration at rest after acute exercise. ${ }^{23,24}$ The results for the PNF group in the present study showed a similar trend as other recently conducted studies that have reported an increase in serum BDNF concentration at rest after the long term endurance training. ${ }^{23,25-27}$ Therefore, it is believed that high intensity exercise will increase resting BDNF concentrations. In particular, Griffin et al. ${ }^{28}$ investigated the effect of acute and long-term cycling exercise in young adults. They found that cognitive function and BDNF were enhanced through acute exercise, and a transient increase in the motor response BDNF expression level was reported in the long-term aerobic exercise group. ${ }^{29-31}$

\section{Conclusion}

We conclude that PNF exercises are may have positive effects on functional outcome and they may enhance neuroplasticity. PNF exercises are specialized and standardized exercises which include neck, trunk, scapula, upper and lower extremity. Along with that audiovisual cues are used, which is not in any other exercises. They can be implemented from first day even in moderate to severe stroke.

\section{Acknowledgements}

This study was supported by the Department of Science and Technology, Project number SR/WOS-A/LS-410/2013, New Delhi, India.

\section{Conflict of interest}

All the authors declare no conflict of interest.

\section{References}

1. Renfro L, Kamino H, Raphael B, et al. Ulcerative lichen planuslike dermatitis associated with hydroxyurea. J Am Acad Dermatol. 1991;24(1):143-145.

2. Mang CS, Campbell KL, Ross CJ, et al. Promoting neuroplasticity for motor rehabilitation after stroke: considering the effects of aerobic exercise and genetic variation on brain-derived neurotrophic factor. Phys Ther. 2013;93(12):1707-1716.

3. Kabat H. Central facilitation; the basis of treatment for paralysis. Perm Found Med Bull. 1952;10(1-4):190-204.

4. Kabat H. Studies on neuromuscular dysfunction. XV. The role of central facilitation in restoration of motor function in paralysis. Arch Phys Med. 1952;33(9):521-533.

5. Irwin OC. Proximodistal differentiation of limbs in young organisms Psychological Review. 1933;40(5):467-477.

6. McGraw MB. Grasping in infants and the proximo-distal course of growth. Psychological Review. 1933;40(3):301-302.

7. Sherrington C. The integrative action of the nervous system. USA: New Haven Yale University Press; 1920.

8. Voss DE. Proprioceptive neuromuscular facilitation. Am J Phys Med. 1967;46(1):838-899.

9. Harvey RL. Improving poststroke recovery: neuroplasticity and taskoriented training. Curr Treat Options Cardiovasc Med. 2009;11(3):251259.

10. Leroux A, Pinet $\mathrm{H}$, Nadeau S. Task-oriented intervention in chronic stroke: changes in clinical and laboratory measures of balance and mobility. Am J Phys Med Rehabil. 2006;85(10):820-830.

11. Rensink M, Schuurmans M, Lindeman E, et al. Task-oriented training in rehabilitation after stroke: systematic review. J Adv Nurs. 2009;65(4):737-754.

12. Bayona NA, Bitensky J, Salter K, et al. The role of task-specific training in rehabilitation therapies. Top Stroke Rehabil. 2005;12(3):58-65.

13. Hubbard IJ, Parsons MW, Neilson C, et al. Task-specific training: evidence for and translation to clinical practice. Occup Ther Int. 2009;16(3-4):175-189.

14. Arabzadeh S, Goljaryan S, Salahzadeh Z, et al. Effects of a task-oriented exercise program on balance in patients with hemiplegia following stroke. Iranian Red Crescent Medical Journal (In Press). 2016:e38429.

15. Singh AK, Chaturvedi P, Maurya PK, et al. Early PNF exercises functional outcome and quality in patients with stroke of urban and rural area in Uttar Pradesh: a prospective community based study. International Journal of Research in Medical Sciences. 2018;6(2):639-644.

16. Kurozumi K, Nakamura K, Tamiya T, et al. BDNF gene-modified mesenchymal stem cells promote functional recovery and reduce infarct size in the rat middle cerebral artery occlusion model. Mol Ther. 2004;9(2):189-197.

17. Schabitz WR, Steigleder T, Cooper-Kuhn CM, et al. Intravenous brainderived neurotrophic factor enhances poststroke sensorimotor recovery and stimulates neurogenesis. Stroke. 2007;38(7):2165-2672.

18. Ploughman M, Windle V, MacLellan CL, et al. Brain-derived neurotrophic factor contributes to recovery of skilled reaching after focal ischemia in rats. Stroke. 2009;40(4):1490-1495

19. Stanne TM, Åberg ND, Nilsson S, et al. Low circulating acute brainderived neurotrophic factor levels are associated with poor long-term functional outcome after ischemic stroke. Stroke. 2016;47(7):19431945.

20. Ferris LT, Williams JS, Shen CL. The effect of acute exercise on serum brain-derived neurotrophic factor levels and cognitive function. $\mathrm{Med} \mathrm{Sci}$ Sports Exerc. 2007;39(4):728-734. 
21. Jeon YK, Ha CH. The effect of exercise intensity on brain derived neurotrophic factor and memory in adolescents. Environ Health Prev Med. 2017;22(1):27.

22. Vega SR, Strüder HK, Wahrmann BV, et al. Acute BDNF and cortisol response to low intensity exercise and following ramp incremental exercise to exhaustion in humans. Brain Res. 2006;1121(1):59-65.

23. Erickson KI, Voss MW, Prakash RS, et al. Exercise training increases size of hippocampus and improves memory. Proc Natl Acad Sci USA. 2011;108(7):3017-3022.

24. Vega SR, Kleinert J, Sulprizio M, et al. Responses of serum neurotrophic factors to exercise in pregnant and postpartum women. Psychoneuroendocrinology. 2011;36(2):220-227.

25. Zoladz JA, Pilc A, Majerczak J, et al. Endurance training increases plasma brain-derived neurotrophic factor concentration in young healthy men. J Physiol Pharmacol. 2008;59 Suppl 7:119-132.

26. Lee SS, Yoo JH, Kang S, et al. The effects of 12 weeks regular aerobic exercise on brain-derived neurotrophic factor and inflammatory factors in juvenile obesity and type 2 diabetes mellitus. J Phys Ther Sci. 2014;26(8):1199-1204.
27. Ruscheweyh R, Willemer C, Krüger K, et al. Physical activity and memory functions: an interventional study. Neurobiol Aging. 2011;32(7):1304-1319.

28. Griffin ÉW, Mullally S, Foley C, et al. Aerobic exercise improves hippocampal function and increases BDNF in the serum of young adult males. Physiol Behav. 2011;104(5):934-941.

29. Kitamura J, Nakagawa H. Visual influence on contact pressure of hemiplegic patients through photoelastic sole image. Arch Phys Med Rehabil. 1996;77(1):14-18.

30. Richard A Schmidt, Lee TD. Motor control and learning: a behavioral emphasis. Champaign, IL, US: Human Kinetics; 2005:537.

31. Warraich Z, Kleim JA. Neural plasticity: the biological substrate for neurorehabilitation. PM \& R. 2010;2(12 Suppl 2): S208-219. 